Abstract: We observe ring structures in high-order harmonics generated in the loose focusing geometry of a semi-infinite gas cell. Phase matching and absorption considerations indicate that the measured signal is restricted to the harmonics generated in the exit plane. We therefore attribute the ring structure to interferences in the single-atom response in the transverse exit plane of the interaction region. Calculations using the stationary phase approximation were used to analyze the contributions of the long and the short trajectories, respectively, and confirm our experimental findings. The simple setup makes quantum path interference measurements feasible without the need for special filtering mechanisms.

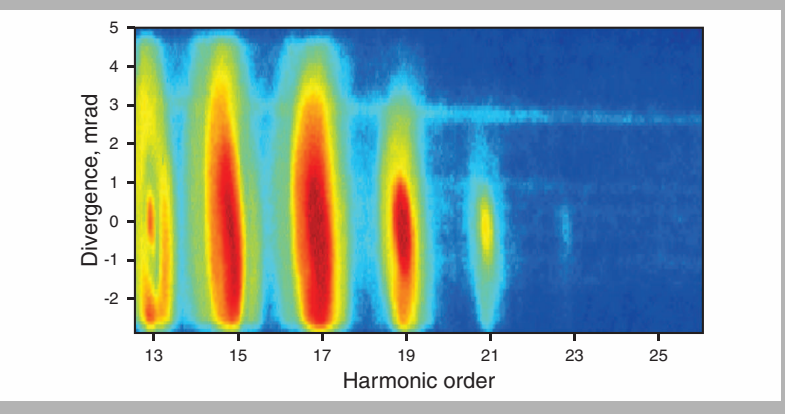

Measured HHG spectrum, generated with an intensity of $0.9 \times 10^{14} \mathrm{~W} / \mathrm{cm}^{2}$. The ring structures in $\mathrm{HH} 13$ originate from the interference of the single-atom responses in the exit plane of the semi-infinite gas cell

\title{
Trajectory interferences in a semi-infinite gas cell
}

\author{
S.M. Teichmann, ${ }^{1, *}$ D.R. Austin, ${ }^{1}$ P. Bates, ${ }^{1}$ S. Cousin, ${ }^{1}$ A. Grün, ${ }^{1}$ M. Clerici, ${ }^{2,3}$ A. Lotti, ${ }^{2,4}$ D. Faccio, ${ }^{2,5}$ \\ P. Di Trapani, ${ }^{2}$ A. Couairon, ${ }^{4}$ and J. Biegert ${ }^{1,6}$ \\ ${ }^{1}$ ICFO-Institut de Ciencies Fotoniques, Mediterranean Technology Park, 08860 Castelldefels (Barcelona), Spain \\ ${ }^{2}$ Nazionale Interuniversitario per le Scienze Fisiche della Materia and Department of Mathematics and Physics, Università \\ dell'Insurbia, 22100 Como, Italy \\ ${ }^{3}$ INRS-EMT, Université du Québec, Varennes, Québec J3X 1S2, Canada \\ ${ }^{4}$ Centre de Physique Théorique, École Polytechnique, Centre National de la Recherche Scientifique, 91128 Palaiseau, France \\ ${ }^{5}$ School of Engineering and Physical Sciences, Scottish Universities Physics Alliance, Heriot-Watt University, Edinburgh, Scotland \\ EH14 4AS, UK \\ ${ }^{6}$ ICREA-Institució Catalana de Recerca i Estudis Avançats, 08010 Barcelona, Spain
}

\section{Introduction}

High-order harmonic generation (HHG) is a common technique for generating extreme ultraviolet (XUV) radiation with pulse durations reaching into the attosecond regime [1-4]. The underlying mechanisms on a microscopic as well as macroscopic scale have been studied extensively and are well understood. In the well-known semi-classical three-step model [5,6] of the single atom response, each emitted photon energy in the plateau can be related to multiple electron trajectories. According to their excursion time in the continuum, the two most prominent trajectories are termed "short" and "long". In the quantum mechanical description [7], electron trajectories translate into quantum paths which accumulate a phase and can show interference properties. Such quantum path interferences (QPI) have attracted significant interest since first demonstrated by A. Zaïr et al. in 2008 [8-14] as they offer insight into the interplay between the microscopic and macroscopic responses of the HHG process by analyzing the far-field interference pattern of the emitted radiation. Moreover, fundamental phenomena of the single atom response, such as yield oscillations with fine wavelength scaling [15], can be attributed to QPI, providing a deeper understanding of the HHG process.

\footnotetext{
* Corresponding author: e-mail: stephan.teichmann@icfo.es
} 


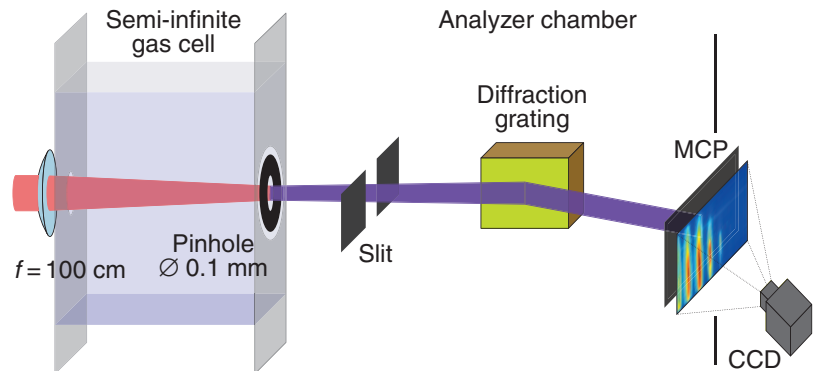

Figure 1 (online color at www.lphys.org) Schematic of the experimental setup (not to scale) with a semi-infinite gas cell. The loose focusing regime is reached using an $f=100 \mathrm{~cm}$ focusing lens. Spectra are acquired as a function of the intensity of the driving field

Here, we study QPI in a new target geometry, namely the semi-infinite gas cell. This type of target is generally characterized by high output fluxes [16,17]. Furthermore, it enables us to study QPI in the very loose focusing regime for the first time. By obtaining far-field spatially resolved spectra, we observe structures which we can relate to the interference of long and short trajectories across the transverse plane of the generating field, modulated by the varying intensity profile of the fundamental field. A simple model based on the stationary phase approximation (SPA) within the strong field approximation (SFA) [7] is used to describe the underlying effects.

\section{Experiment}

High-order harmonic generation is realized in a semiinfinite gas cell using a commercial, amplified Ti:Sapphire system operating at $5 \mathrm{kHz}$ and delivering $30 \mathrm{fs}$ pulses at $800 \mathrm{~nm}$. A schematic of the experimental setup is shown in Fig. 1. Using an $f=100 \mathrm{~cm}$ focusing lens placed $\approx 97 \mathrm{~cm}$ from the pinhole, intensities of $\approx 1 \times 10^{14} \mathrm{~W} / \mathrm{cm}^{2}$ are produced. The pulses are not CEP-stabilized, which is unproblematic because we are investigating the interference of quantum paths of the same half cycle [8]. The target chamber is filled with 75 mbar of argon and terminated by a $100 \mu \mathrm{m}$ pinhole, causing an abrupt transition to vacuum. As shall be discussed below, this geometry does not favor either the long or the short quantum paths. In the analyzer chamber the generated XUV radiation is dispersed spectrally by a reflective flat-field grating (1000 lines/mm, Hitachi), positioned $\approx 1035 \mathrm{~mm}$ downstream from the pinhole, and impinges upon a micro-channel plate (MCP, Hamamatsu) with a fluorescent back screen. This signal is imaged onto a CCD camera, enabling us to resolve the harmonic radiation both in angle and frequency in the far field, i.e. in the $(\omega, \theta)$ domain.

Observed spectra for several intensities of the fundamental field between $0.6-1.2 \times 10^{14} \mathrm{~W} / \mathrm{cm}^{-2}$ are shown
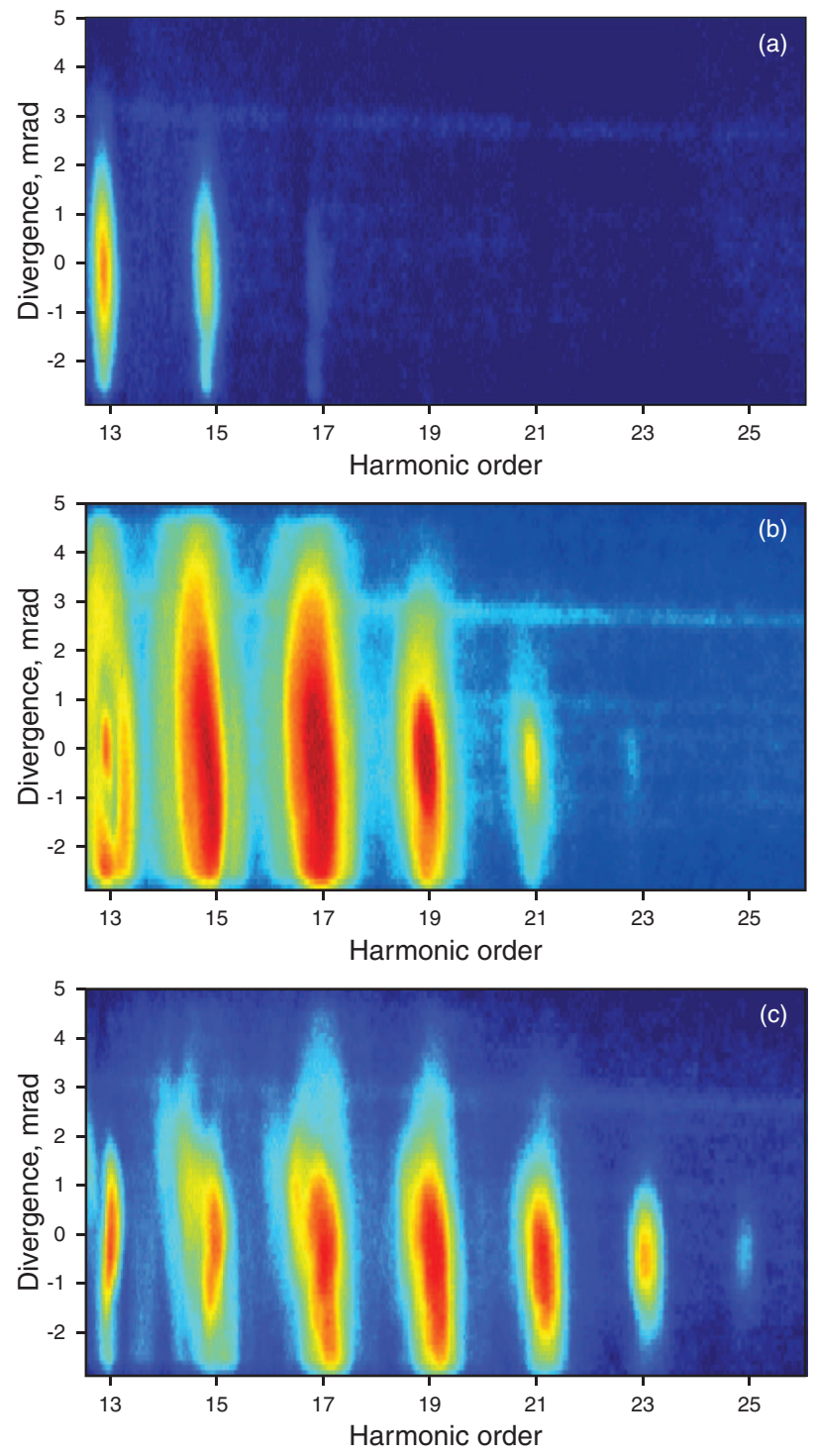

Figure 2 (online color at www.lphys.org) Harmonic spectra (normalized) generated in 75 mbar of argon with (a) $I=0.6 \times 10^{14} \mathrm{~W} / \mathrm{cm}^{2}, \quad$ (b) $I=0.9 \times 10^{14} \mathrm{~W} / \mathrm{cm}^{2}$, and (c) $I=1.2 \times 10^{14} \mathrm{~W} / \mathrm{cm}^{2}$

in Fig. 2. We find the expected increase of the cut-off with increasing driving intensity, generating harmonic orders up to $\mathrm{HH} 25$.

HH13 in Fig. 2b shows a central peak, surrounded by a minimum and a following maximum, forming a ring-like structure in angle-frequency space. As shall be discussed in Sec. 3.1, these structures can be attributed to quantum path interferences of the long and the short trajectories which originate from the intensity profile of the generating beam.

Interestingly, we do not observe the quantum path interference pattern in higher orders of the same measure- 


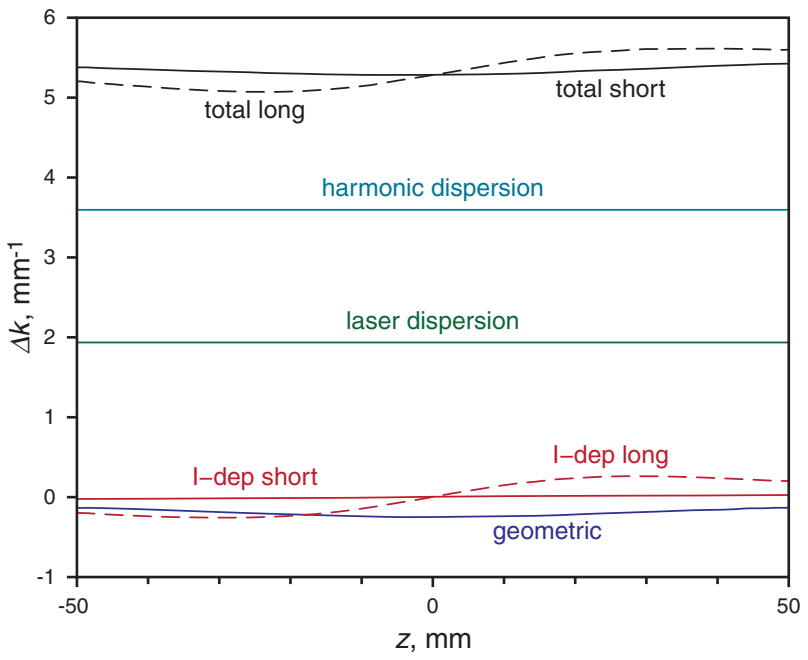

Figure 3 (online color at www.lphys.org) The different phase mismatch contributions on axis, calculated for $\mathrm{HH} 13$ and the used experimental parameters. The total phase mismatch $\Delta k$ for the long and short quantum paths, respectively, are termed "total long" and "total short". See the main text for definitions of the individual contributions

ment. Neither do they appear in any of the harmonics at different laser intensities as shown in Fig. 2a and Fig. 2c, indicating that particular experimental parameters are required to observe QPI. These results shall be discussed below.

\section{Analysis}

In this section we discuss the origin of the observed farfield ring structures by relating general features of the single atom response (Sec. 3.1) to the particular parameters of our experiment (Sec. 3.2 and Sec. 3.3). We find in Sec. 3.2 that for our parameters macroscopic quantum path selection is weak, particularly so for low order harmonics such as HH13. Based on this we investigate the single atom response in the transverse exit plane of the semi-infinite gas cell in Sec. 3.3, showing that the observed interference structures are created dominantly in this part. The discussion is based on the concepts of the strong field approximation (SFA) [7] and the stationary phase approximation (SPA) which facilitate the assignment of emission times to harmonic frequencies through classical mechanics of charged particles in a sinusoidally varying laser field.

\subsection{Quantum path interference mediated by the intensity profile}

We shall briefly review QPI, limiting the discussion to the interference between the long and the short trajecto- ries only. The phase of the dipole amplitude is characterized by a dependence on the intensity of the driving field [18]. This phase manifests in a "chirp", or shift of the instantaneous frequency, and a divergence of the harmonic signal in the far field, i.e. the $(\omega, \theta)$ domain. These displacements are taken relative to the signal generated at peak intensity, which appears on axis in this domain. The intensity-dependent dipole phase, however, is different for the short and long trajectories, such that for a given intensity value of the driving field in the near field,i.e. the $(t, x)$ domain, their respective chirps and divergences are not equal. Within a Gaussian intensity profile in $(t, x)$ the phase difference between the two trajectories might become zero, though, making constructive interference in the $(\omega, \theta)$ domain detectable [13]. Here, we investigate such interference structures of the single atom response in the loose focusing regime.

\subsection{Phase matching and absorption}

In order to relate the general QPI features discussed in Sec. 3.1 to the particular conditions of the experiment, it is instructive to analyze the effects of phase matching as well as absorption on the propagation of the generated field [19]. To do so, we describe the growth of the generated field $E(z)$ in the reference frame of the source as

$\frac{d E}{d z}=g-(i \Delta k+\alpha) E$

which is valid when coherence and absorption lengths are small compared to other characteristic lengths of the system, e.g. the target size and the Rayleigh range. We shall show below that this is the case here. In Eq. (1), $\Delta k$ is the phase mismatch between the generated field and the atomic dipole while $\alpha$ is the absorption coefficient. For large values of $z$, the solution approaches $g L_{\text {eff }} \exp \left(i \phi_{\text {eff }}\right)$ where [19]

$L_{e f f}=\frac{1}{\sqrt{\alpha^{2}+\Delta k^{2}}}, \quad \phi_{e f f}=\tan ^{-1}\left(\frac{\Delta k}{\alpha}\right)$.

The effective generation length $L_{\text {eff }}$ describes the distance over which the harmonic signal is generated and is a function of the phase mismatch and the absorption. Only if $L_{e f f}$ is similar for two trajectories can QPI occur between them. The effective phase $\phi_{e f f}$ is the phase difference between the macroscopic response and the dipole response introduced by propagation. We shall show that both $L_{e f f}$ and $\phi_{\text {eff }}$ are comparable for the long and the short trajectories, meaning that the measured signal is close to that of the single atom response.

The overall phase mismatch $\Delta k$ between the fundamental driving field and the generated field is given by $\Delta k=\Delta k_{\text {geom }}+\Delta k_{H H}+\Delta k_{\text {las }}+\Delta k_{\text {int }}$. Here, $\Delta k_{\text {geom }}$ is the geometric factor, or Gouy phase shift, of a propagating Gaussian beam; $\Delta k_{H H}$ and $\Delta k_{\text {las }}$ describe the dispersion of the harmonics and the laser field, respectively, 

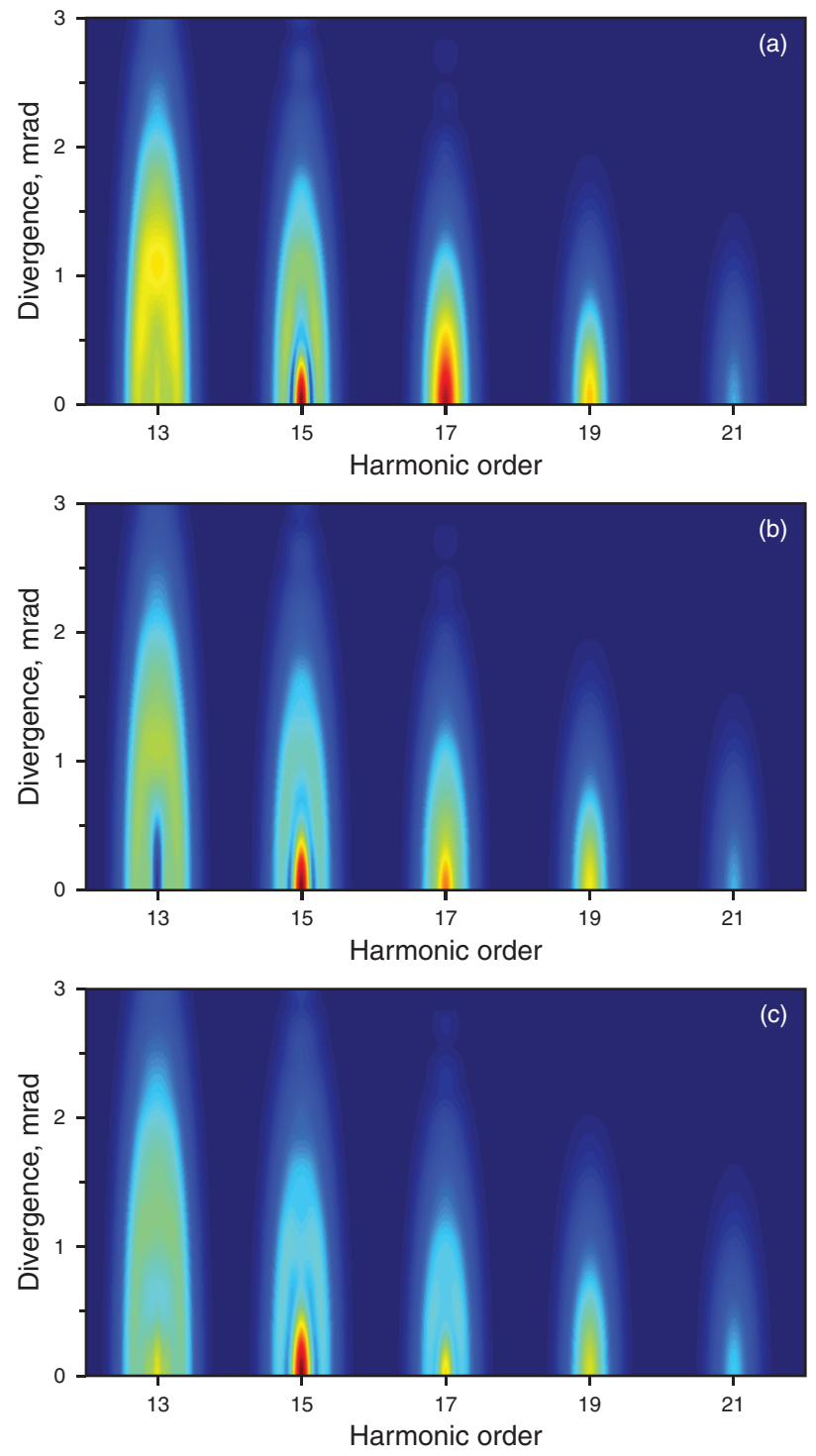

Figure 4 (online color at www.lphys.org) Simulation results with intensities of (a) $0.86 \times 10^{14} \mathrm{~W} / \mathrm{cm}^{2}$, (b) $0.90 \times 10^{14} \mathrm{~W} / \mathrm{cm}^{2}$, and (c) $0.96 \times 10^{14} \mathrm{~W} / \mathrm{cm}^{2}$. The far field QPI ring structures observed experimentally in $\mathrm{HH} 13$ at an intensity of $0.90 \times 10^{14} \mathrm{~W} / \mathrm{cm}^{2}$ are reproduced in a) and c), indicating the strong intensity dependence of interferences of the single-atom responses in the transverse exit plane of the generation chamber

taken with respect to propagation in vacuum; $\Delta k_{i n t}$ results from the intensity-dependence of the atomic dipole response.

We evaluate the different phase mismatch contributions for $\mathrm{HH} 13$ and the experimental parameters in Fig. 3. Shown are the contributions on axis for the long and the short trajectories along the propagation direction around the focus. The dominating phase mismatch contributions are the positive dispersion terms which are much larger in magnitude than the negative geometric and intensitydependent contributions, resulting in $l_{c o h}=\pi / \Delta k \ll z_{R}$ (the Rayleigh length is given by $z_{R}=\pi w_{0}^{2} / \lambda$, where $w_{0}$ is the beam waist and $\lambda$ the laser wavelength). Differences in the phase mismatch between the long and the short trajectories stem from the intensity-dependent phase term which is equal at the focus.

Evaluating Eq. (2) with the calculated phase mismatch values $\Delta k$ and the absorption coefficient $\alpha=3.4 \mathrm{~mm}^{-1}$, we find that in the focal region $L_{e f f}$ is comparable to $l_{c o h}$, while both lengths are shorter than $z_{R}$. The measured signal of $\mathrm{HH} 13$ is thus dominated by the single atom response at the exit plane of the semi-infinite gas cell. Furthermore, the intensity-dependent dipole phase shown in Fig. 3 only has a negligible impact on $\Delta k$ such that $L_{e f f, \text { short }} \approx L_{\text {eff,long }}$, indicating that neither trajectory is preferentially selected macroscopically. Moreover, we find with the calculated phase mismatch values that in the focal region $\phi_{\text {eff,short }} \approx \phi_{\text {eff,long }}$, suggesting that the long and short trajectories are in phase in the transverse exit plane. As a result, the macroscopic high harmonics signal is similar to that of the single atom response for both trajectories and since their effective phases are similar, QPI can occur in the transverse exit plane. This is in contrast to the QPI measurements by F. Schapper et al. [13] where off-axis spatial filtering was required.

Our loose focusing geometry, resulting in a short effective generation length and a negligible difference in the effective phases of the long and the short trajectories, thus enables us to detect QPI from the transverse exit plane of the semi-infinite gas cell. Furthermore, measuring harmonic orders around HH13 simplifies the detection of QPI structures due to their relatively high divergence.

\subsection{Transverse single-atom response}

To test the discussed dependence of QPI structures on phase matching and absorption parameters, we performed numerical simulations. The fundamental field is propagated linearly to the exit plane of the semi-infinite gas cell where the light-matter interaction is simulated using the strong-field approximation (SFA)[7]. To calculate the dipole response in the SFA we use a stationary phase approximation in momentum, birth time and return time, allowing us to disentangle the contributions of different quantum paths. The far-field signal is obtained by applying a Hankel transform in space and a Fourier transform in time to the dipole response in the near field. Our simulations reveal that the visibility of QPI structures for a given harmonic order in the far field, i.e. the contrast ratio of the ring structure, crucially depends on the used intensity, confirming our experimental observations. A qualitatively reasonable match for the structures in $\mathrm{HH} 13$ is obtained in the simulation using an intensity in the range of $0.86-$ $0.96 \times 10^{14} \mathrm{~W} / \mathrm{cm}^{2}$, see Fig. 4, which is in good agreement with the experimentally determined $0.9 \times 10^{14} \mathrm{~W} / \mathrm{cm}^{2}$, see 
Fig. 2b. This supports our analysis that the measured HHG signal is produced at the exit plane of the target chamber with QPI structures originating from interferences of the single-atom responses in the transverse plane.

\section{Conclusion}

Recently, several different interesting aspects of strongfield physics [20] and high harmonic generation in particular have been investigated [21-25]. In this work, we investigate interference structures of the short and long quantum paths in HHG in a semi-infinite gas cell. Using an intensity of $0.9 \times 10^{14} \mathrm{~W} / \mathrm{cm}^{2}$ in the loose focusing regime we observe ring structures in $\mathrm{HH} 13$ of the fundamental $800 \mathrm{~nm}$. By analyzing phase matching and absorption characteristics of our experiment, we show that longitudinal phase mismatch and absorption restrict the observed signal to the harmonics generated at the exit plane of the target chamber. The observed interference structures are thus a consequence of the interferences of the single-atom responses in the transverse exit plane, as confirmed by numerical simulations.

We note that the femtosecond laser system used in this experiment offers the opportunity to study various other interesting fields, among which are the generation of optical frequencies in the telecommunication band [26], terahertz emission from filaments $[27,28]$ and filamentary selfcompression [29].

Acknowledgements Six authors (A.G., D.R.A., J.B., P.B., S.C., and S.M.T.) acknowledge partial support from the Spanish Ministry of Education and Science through its Consolider Program Science (SAUUL CSD 2007-00013), as well as through Plan Nacional (FIS2008-06368-C02-01). Funding from LASERLABEUROPE, grant agreement 228334, is gratefully acknowledged. One author (D.R.A.) acknowledges support from a Marie Curie Intra-European Fellowship (project 276556-BAXHHG). Four authors (A.L., D.F., M.C., and P.DT.) acknowledge financial support from the Consorzio Nazionale Interuniversitario per le Scienze della Materia, project Innesco.

\section{References}

[1] P. Agostini and L.F. DiMauro, Rep. Prog. Phys. 67, 813 (2004).

[2] P.B. Corkum and F. Krausz, Nat. Phys. 3, 381 (2007).

[3] F. Krausz and M. Ivanov, Rev. Mod. Phys. 81, 163 (2009).

[4] M. Nisoli and G. Sansone, Prog. Quantum Electron. 33, 17 (2009).

[5] P.B. Corkum, Phys. Rev. Lett. 71, 1994 (1993).

[6] K.C. Kulander, K.J. Schafer, and J.L. Krause, in: B. Piraux, A. L'Huillier, and K. Rzążewski (eds.), SuperIntense Laser-Atom Physics, NATO Science Series, B Physics, vol. 316 (Plenum Press, New York, 1993), p. 95.
[7] M. Lewenstein, Ph. Balcou, M.Yu. Ivanov, A. L'Huillier, and P.B. Corkum, Phys. Rev. A 49, 2117 (1994).

[8] A. Zaï, M. Holler, A. Guandalini, F. Schapper, J. Biegert, L. Gallmann, U. Keller, A.S. Wyatt, A. Monmayrant, I.A. Walmsley, E. Cormier, T. Auguste, J.P. Caumes, and P. Salières, Phys. Rev. Lett. 100, 143902 (2008).

[9] H. Xu, H. Xiong, Z.A. Zeng, Y.X. Fu, J.P. Yao, R.X. Li, Y. Cheng, and Z.Z. Xu, Phys. Rev. A 78, 033841 (2008).

[10] E. Brunetti, R. Issac, and D.A. Jaroszynski, Phys. Rev. A 77, 023422 (2008).

[11] M. Holler, A. Zaïr, F. Schapper, T. Auguste, E. Cormier, A. Wyatt, A. Monmayrant, I.A. Walmsley, L. Gallmann, P. Salières, and U. Keller, Opt. Express 17, 5716 (2009).

[12] T. Auguste, P. Salières, A.S. Wyatt, A. Monmayrant, I.A. Walmsley, E. Cormier, A. Zaïr, M. Holler, A. Guandalini, F. Schapper, J. Biegert, L. Gallmann, and U. Keller, Phys. Rev. A 80, 033817 (2009).

[13] F. Schapper, M. Holler, T. Auguste, A. Zaïr, M. Weger, P. Salières, L. Gallmann, and U. Keller, Opt. Express 18, 2987 (2010).

[14] J.E. Kruse, P. Tzallas, E. Skantzakis, and D. Charalambidis, Phys. Rev. A 82, 033438 (2010).

[15] K. Schiessl, K.L. Ishikawa, E. Persson, and J. Burgdörfer, Phys. Rev. Lett. 99, 253903 (2007).

[16] J. Peatross, J.R. Miller, K.R. Smith, S.E. Rhynard, and B.W. Pratt, J. Mod. Opt. 51, 2675 (2004).

[17] D.S. Steingrube, T. Vockerodt, E. Schulz, U. Morgner, and M. Kovačev, Phys. Rev. A 80, 043819 (2009).

[18] M. Lewenstein, P. Salières, and A. L'Huillier, Phys. Rev. A 52, 4747 (1995).

[19] E. Constant, D. Garzella, P. Breger, E. Mével, Ch. Dorrer, C. Le Blanc, F. Salin, and P. Agostini, Phys. Rev. Lett. 82, 1668 (1999).

[20] I.A. Burenkov, A.M. Popov, O.V. Tikhonova, and E.A. Volkova, Laser Phys. Lett. 7, 409 (2010).

[21] M. Negro, C. Vozzi, K. Kovacs, C. Altucci, R. Velotta, F. Frassetto, L. Poletto, P. Villoresi, S. De Silvestri, V. Tosa, and S. Stagira, Laser Phys. Lett. 8, 875 (2011).

[22] J.A. Pérez-Hernández, J. Ramos, L. Roso, and L. Plaja, Laser Phys. 20, 1044 (2010).

[23] C. Vozzi, F. Calegari, F. Frassetto, M. Negro, L. Poletto, G. Sansone, P. Villoresi, M. Nisoli, S. De Silvestri, and S. Stagira, Laser Phys. 20, 1019 (2010).

[24] R.A. Ganeev, Laser Phys. 21, 25 (2011).

[25] A. Pirri, E. Sali, C. Corsi, M. Bellini, S. Cavalieri, and R. Eramo, Laser Phys. 20, 1119 (2010).

[26] Y.-J. Kim, B.J. Chun, Y. Kim, S. Hyun, and S.-W. Kim, Laser Phys. Lett. 7, 522 (2010).

[27] T.-J. Wang, C. Marceau, S. Yuan, Y. Chen, Q. Wang, F. Théberge, M. Châteauneuf, J. Dubois, and S.L. Chin, Laser Phys. Lett. 8, 57 (2011).

[28] T.-J. Wang, J.-F. Daigle, Y. Chen, C. Marceau, F. Théberge, M. Châteauneuf, J. Dubois, and S.L. Chin, Laser Phys. Lett. 7, 517 (2010).

[29] C. Brée, A. Demircan, and G. Steinmeyer, Laser Phys. 21, 1313 (2011). 